\title{
Perfil lipídico en recién nacidos normales de ambos sexos
}

\author{
Víctor Casanueva E. ${ }^{1 ;}$ Ximena Cid C..; Cristián Milos G. ${ }^{3}$; María Teresa Chiang S. ${ }^{*}$; \\ Carolina Lama L. ${ }^{1}$; Fernando Heredia J. ${ }^{2}$; Aldo Bancalari M. ${ }^{5}$
}

\section{Serum lipids in healthy newborn infants}

This study was undertaken to delermine the concentralion and distribulion of serum lipids in healthy newborn intants at Concepcion city, in southern Chile. An umbilicol cord blood sample was taken immedialely alter birlh from 220 newborn infants. Mean serum lipid concentrations (male/temole/total sample) were: told choleslerol $=57.2 / 63.4$ / $60.4 \mathrm{mg} / \mathrm{dl} ; \mathrm{HDLC}=24.5 / 29.5 / 26.8 \mathrm{mg} / \mathrm{dl} ; \quad$ LCtC $=27.2 / 29.2 / 28.3 \mathrm{mg} / \mathrm{dl} ;$;riglycerides $=24.4 / 21.8 / 23.9$ $\mathrm{mg} / \mathrm{dl}$. There were signilicant differences between mole and female serum concentrations of tatal cholesterol, $\mathrm{HDL} \cdot \mathrm{C}$ ip $<0.001\}$ and triglycerides $\mid p<0.01\}$. In the whole sample, serum lipids 5 th $/ 50$ h $/ 95$ th percentiles were: total cholesterol $=37.4 / 57.2 / 85.1 \mathrm{mg} / \mathrm{dl} ; H \cdot D l C=15.0 / 20.8 / 34.0 \mathrm{mg} / \mathrm{dl}: L \mathrm{LLC}=10.0 / 15.7 / 28.8 \mathrm{mg} / \mathrm{dl}$ and triglycerides $=10.0 / 20.7 / 48.0 \mathrm{mg} / \mathrm{dl}$, being similor to those reported from oher countries and significanily lower than in older children and adults. Serum tolal cholestesol showed high comelation rates wilh $\mathrm{HOL} \cdot \mathrm{C} \mid \mathrm{r}=0.70 \mathrm{H})$ and $L \mathrm{DL} \cdot \mathrm{C}$ $i=0.775$. If maximal normal point lor total serum cholesterol is set of $<95 \mathrm{mg} / \mathrm{dl}$ and that of serurn triglycerides at $<05$ $\mathrm{mg} / \mathrm{dl}$, then $0.9 \%$ of our samples show abnormally high cholesterd ond $1.8 \%$ abnormolly high Iriglycerides concenthation.

[Kay words: serum lipids, newborn, cond blood.]

En Chile, como en otros países, las enfermedades cardiovasculares constituyen la primera causa de mortalidad general'. En la actualidad, se tiende a establecer medidas preventivas en la niñez ${ }^{2}$, por considerar que la aterogénesis tiene su origen en este período, en el que además se establecen el estilo de vida y los hábitos de alimentación. Si la hipercolesterolemia familiar es uno de los errores congénitos metabólicos mấs comunes ${ }^{3}$, entonces su detección temprana permitiría aplicar medidas preventivas a los afectados.

1. Departamento de Pediatría, Facultad de Mcdicina, Universidad de Concepción.

2. Bioquímico. Laboratorio Central, Hospital Regional Guillermo Grant Benavente, Servicio de Salud, Concepcion.

3. Químico Farmacéulico. Departamento de Bioquímica Clínica e Inmunología, Facultad de Famacia, Universidad de Concepcion.

4. Bioquímico. Laboratorio de Ergonomía, Departamento de Fisiopatología, Facultad de Ciencias Biológicas, Lniversidad de Concepción.

5. Laboratorio Central, Hospital Regional Guillemo Grant Benavente, Servicio de Salud, Concepeion.

Proyecto financiado por la Dirección de Investigación. Universidad de Concepción, $\mathrm{N}^{\mathrm{r}} 9288$ 12-t.
Este estudio se diseñó con el objeto de describir la concentración y distribución de los lípidos séricos en recién nacidos sanos en nuestro medio, para facilitar estudios ulteriorcs sobre el valor predictivo de la concentración de las diferentes lipoproteínas en el plasma.

\section{Material y Método}

Se hicieron mediciones en la sangre de cordón unbilical de 220 recién nacidos de ambos sexos (103 niños y 117 ninas) provenientes de las unidades de meonatología del Hos. pital Clúnico Guillemo Grant Benavente y de la Clínica Francesa, ambas de Concepción. Los recién nacidos fueron seleccionados de acuerdo a los siguientes criterios: odad gestacional entre 38 y 41 semarlas; peso al nacer adecuado a edad gestacional (AEG); A pgar al minuo mayor o igual a 7; ausencıa de morbilidad perinatal o malformaciones congénitas; aısencia de enfermedades máemas, incluídas hipencusión arterial, diabetes, hipotiroidismo, hipertiroidismo y colestasis. La sangre de cordón se chtuvo inmediatamente después del parto y correspondió a la que nomalmente sc extrae para determinación de VDRL en todos los recién nacidos. Parte de ella fue utilizada para medir la concentración sérica de los lípidos, por lo que no significo extracción adicional de sangre. Las mediciones se hicieron mediante métodos enzimáticos del Laboratorio Bochringer, Mannheim, descritos en una publi- 
cación previa4. El análisis estadístico para verificar diferenciss entre grupos se realizb utilizando prueba I de Swdent, aceptándose como significativo $p<0,05$.

\section{Resultados}

En la figura se muestra la disuribución de las concentraciones de colesterol total, colesterol unido a lipoproteínas de alta densidad (C-HDL), colesterol unido a lipoproteínas de baja densidad (C-LDL) y triglicéridos, por sexo y en el conjunto de la muestra ${ }^{15}$. Los promedios y las desviaciones estándar correspondientes se describen en la tabla 1 y los percentiles en la tabla 2 .

En el caso del colesterol total, las concentraciones en el suero fueron $57,2 \pm 11,7$ y $63,4 \pm$ $13,2 \mathrm{mg} / \mathrm{dl}$ para niños y niñas respectivamente, con una diferencia estadísticamente significativa $(p<0,001)$. La del conjunto fue $60,4 \pm 13,7$ $\mathrm{mg} /$ dl. Los percentiles 90 y 95 fueron 71,3 y 77,4 $\mathrm{mg} / \mathrm{dl}$ en varones; 80,0 y $85,8 \mathrm{mg} / \mathrm{dl}$ en niñas $y$ 78,3 y $85,1 \mathrm{mg} / \mathrm{d}$ l en el conjunto, respectivamente. Si se considera a los valores entre los percentiles 5 y 95 (90\% de la población) como márgenes de referencia, nuestra población estaria entre 37,4 y $85,1 \mathrm{mg} / \mathrm{dl}$, siendo las cifras extremas de colesterol total 36 y $99 \mathrm{mg} / \mathrm{dl}$. La distribución de las concentraciones de colesterol ligado a lipoproteínas de alta densidad (C-HDL) fueron, en los nifos $24,5 \pm 7,0 \mathrm{mg} / \mathrm{dl}$, en las niñas $29,5 \pm 8,6$ $\mathrm{mg} / \mathrm{dl}$, điferencias que también fueron estadísticamente significativas ( $p<0,0001$ ). Para el conjunto de recién nacidos el promedio de la concentración de esta fracción fue $26,8 \pm 8,4 \mathrm{mg} /$ di. En $90 \%$ de los nifíos estudiados las concentraciones séricas de ella estaban entre 15 y $34 \mathrm{mg} / \mathrm{dl}$. Los promedios y desviaciones estándar del colesterol unido a lipoproteínas de baja densidad (C-LDL) fueron $27,2 \pm 8,6$ en varones y $29,2 \pm 8,6$ en las niffas, sin diferencias estadísticamente significativa enire ellos, con $90 \%$ de los valores entre 10,0 y $28,8 \mathrm{mg} / \mathrm{dl}$. Se observó una buena correlación tanto entre colesterol total y C-HDL $(r=0,70)$, como entre aquél y C-LDL $(r=0,78)$. La distribución de los uriglicéridos se describe en la figura. Las concentraciones plasmáticas de triglicéridos fueron $26,4 \pm 14,5 \mathrm{mg} / \mathrm{dl}$ y $21,8 \pm 9,9$ mg/dl para niffos y nif̃as respectivamente, diferencia que es estadisticamente significativa ( $<<0,01$ ); el promedio de ellas para el total de las muestras fue de $23,9 \pm 12,6 \mathrm{mg} / \mathrm{dl}$ y $90 \%$ se ubicaban entre 10,0 y $48,0 \mathrm{mg} / \mathrm{dl}$ (figura, tablas 1 y 2 ).
Tabla 1

Concentración sérica de colesterol total (CT), C-HDL, C-LDL y triglicéridos

(TG) en 220 recién nacidos,

por sexo y total

\begin{tabular}{lccc}
\hline $\begin{array}{l}\text { Variable } \\
\text { (mg/dl) }\end{array}$ & $\begin{array}{c}\text { Niños } \\
\mathbf{n}: 116\end{array}$ & \multicolumn{1}{c}{$\begin{array}{c}\text { Niñas } \\
\mathbf{n}: \mathbf{1 0 4}\end{array}$} & $\begin{array}{c}\text { Grupo total* } \\
\mathbf{n}: \mathbf{2 2 0}\end{array}$ \\
\hline CT & $57,2 \pm 11,7$ & $63,4 \pm 13,2^{* *}$ & $60,4 \pm 13,7$ \\
C-HDL & $24,5 \pm 7,0$ & $29,5 \pm 8,6^{* *}$ & $26,8 \pm 8,4$ \\
C-LDL & $27,2 \pm 8,6$ & $29,2 \pm 8,6$ & $28,3 \pm 9,5$ \\
TG & $24,4 \pm 14,5$ & $21,8 \pm 9,9 * * *$ & $23,9 \pm 12,6$ \\
\hline
\end{tabular}

* : grapo total considera ambos sexos

**: : $(p<0,001)$ cntre niños $y$ niñas

*** : ( $p<0,01)$ entre niños y niñas

CT : colesterol total

TG : triglicéridos

HD : lipoproteinas de alta densidad

LD : lipoproteinas de baja densidad.

Tabla 2

Percentil 5, 10, 50, 90 y 95 de los lípidos séricos en recién nacidos niños, niñas y grupo total expresados en $\mathrm{mg} / \mathrm{dl}$

\begin{tabular}{llllll}
\hline \multicolumn{5}{c}{} & \multicolumn{5}{c}{ P e r e n t l } \\
& 5 & 10 & 50 & 90 & 95 \\
\hline Niños & & & & & \\
CT & 33,5 & 40,5 & 55,5 & 71,3 & 77,4 \\
C-HDL & 13,3 & 15,3 & 23,0 & 34,1 & 37,7 \\
C-LDL & 12,9 & 16,4 & 25,9 & 38,1 & 42,9 \\
TG & 10,0 & 11,7 & 22,3 & 45,3 & 56,0 \\
Niñas & & & & & \\
CT & 41,1 & 44,5 & 64,2 & 80,0 & 85,8 \\
C-HDL & 13,3 & 16,1 & 28,2 & 42,1 & 46,0 \\
C-LDL & 12,7 & 20,8 & 29,1 & 41,7 & 45,7 \\
TG & 10,0 & 11,6 & 21,1 & 34,7 & 44,2 \\
Total & & & & & \\
CT & 37,4 & 42,2 & 57,2 & 78,3 & 85,1 \\
C-HDL & 15,0 & 15,8 & 20,8 & 30,0 & 34,0 \\
C-LDL & 10,0 & 10,7 & 15,7 & 25,0 & 28,8 \\
TG & 10,0 & 11,3 & 20,7 & 40,0 & 48,0 \\
\hline
\end{tabular}

CT:colesterol total TG: triglicéridos HDL.ilipoproteínas de alta densidad LDL:Lipoprateínas de baja densidad.

\section{Comentario}

En recién nacidos las concentraciones de colesterol total, C-HDL, C-LDL y triglicéridos se distribuyen en márgenes considerablemente 
OISTRIBUCHON FRECUENCIA COLESTEROL

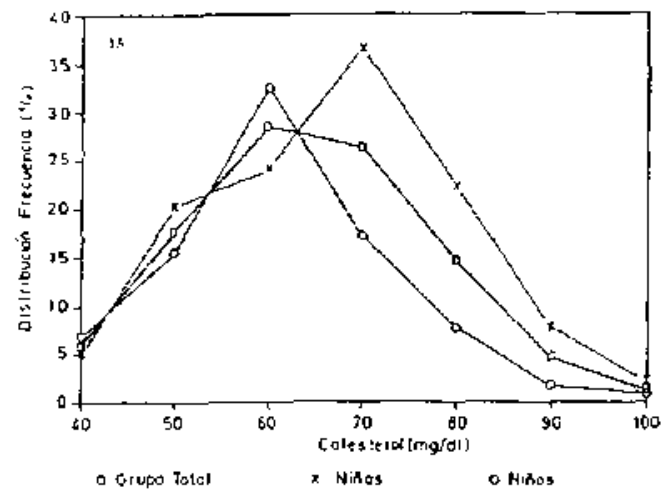

DISTRIBUCION FRECUENCIA C-LDL

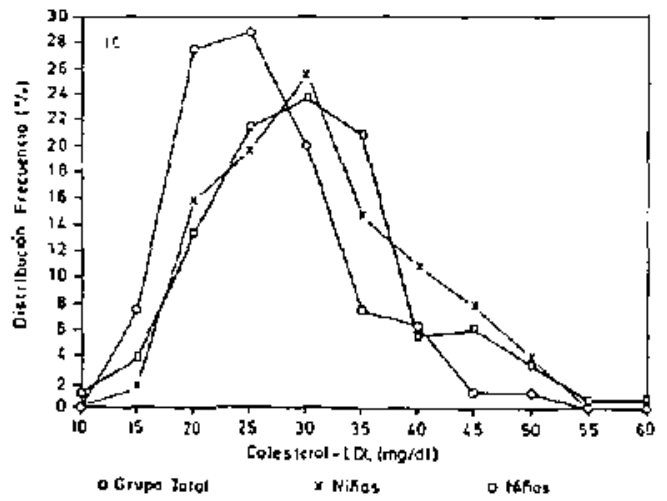

DISTRIBUCION FRECUENCIA C-HDL

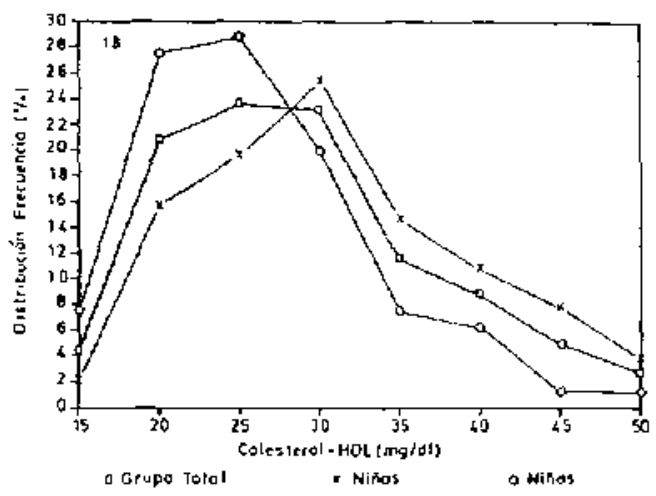

DISTRIBUCION FRECUENCIA IRIGLICERIDOS

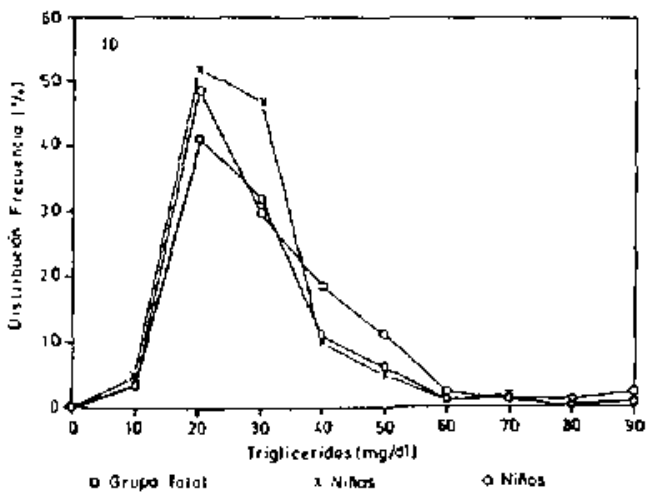

Figura 1: Distribución de frecuencia de colesterol, colesterol HDL. colesterol LDL y triglcéridos de niños, niñas y grupo total.

menores que en niños y adultos, las concentraciones promedio de lípidos y lipoproteínas séricas obtenidas en nuestro estudio son comparables con las descritas por otros autores ${ }^{6-15}$.

Se postula que los bajos niveles de colesterol total y especialmente de C-LDL que se observan al nacer se deberían a mayor cantidad de receptores de C-LDL que en el adulto ${ }^{16}$. En apariencia los receptores de C-LDL están maduros y en pleno funcionamiento en el feto y el recién nacido ${ }^{17}$. En el feto, el colesterol se usa principalmente como C-LDL para la producción de esteroides, como los de origen suprartenal. Al nacer, la demanda de C-LDL disminuye, por este motivo aumentan, en los primeros días de vida, las concentraciones de colesterol total y $C-\mathrm{LDL}^{\mathrm{LB}}$. Esta variación de las lipoproteínas después del nacimiento se de- bería también al estrés propio del parto, que estimularía la lipogénesis en los recién nacidos, pasando los lípidos a constituir su principal fuente de energía ${ }^{10}$.

Si se consideran como concentraciones límites de colesterol total $95 \mathrm{mg} / \mathrm{dl}$ y de triglicéridos $65 \mathrm{mg} / \mathrm{dl}^{19}$, entonces $0,9 \%$ de nuestros recién nacidos exceden las primeras y $1,8 \%$ las últimas. Para detectar hiperlipidemia no basta, sin embargo, una determinación de lipidos, por las variaciones propias de la primera semana de vida. No obstante, cuando aparecen valores alterados es importante realizar seguimiento y estudio de la familia, con el fin de descartar o pesquisar un trastorno, con lo que podriamos prevenir, cn etapas muy tempranas de la vida, una posible enfermedad cardiovascular durante la vida adulta. 


\section{Resumen}

Este estudio se diseñó con el objeto de determinar la concentración y distribución de lipidos séricos en recién nacidos sanos de nuestro medio, teniendo en consideración que en Chile, como sucede en la mayoría de los países desarrollados, las enfermedades cardiovasculares constituyen la primera causa de mortalidad general y su prevención debe comenzar en la niñez. Se obtuvo muestra de sangre de cordón de 220 recién nacidos de ambos sexos, inmediatamente después del parto. Los resultados obtenidos fueron (ninos/ninas/total de la muestra): colesterol total $=57,2 / 63,4 / 60,4 ; \mathrm{C}-\mathrm{HDL}=24,5 / 29,5 / 26,8$; $\mathrm{C}$-LDL $=27,2 / 29,2 / 28,3$; triglicéridos $=24,4 / 21,8$ / $23,9 \mathrm{mg} / \mathrm{dl}$. Hubo diferencias significativas entre los sexos, $(p<0,001)$ en las concentraciones séricas de colesterol total, C-HDL $(\mathrm{p}<0,001)$ y triglicéridos $(p<0,01)$. Considerando todos los recién nacidos estudiados, los percentiles p5/p50/ p95 de las concentraciones fueron: colesterol total $=37,4 / 57,2 / 85,1 ; \mathrm{C}-\mathrm{HDL}=15,0 / 20,8 / 34,0$; $\mathrm{C}-\mathrm{LDL}=10,0 / 15,7 / 28,8$ y triglicéridos $=10,0 \%$ $20,7 / 48,0 \mathrm{mg} / \mathrm{dl}$, similares a las de otros países y significativamente menores que las de niños y adultos. Se observó una correlación alta entre los valores de colesterol total y C-HDL $(r=0,701)$ y entre aquél y C-LDL $(r=0,775)$. Al considerar como límites normales valores de colesterol $<95$ y triglicéridos $<65 \mathrm{mg} / \mathrm{dl}$, enconiramos que $0,9 \%$ de la muestra presenta alterado su nivel de los primeros y $1,8 \%$ de los segundos.

(Palabras clave: colesterol, lipoproteínas séricas, tecién nacidos.)

\section{Referencias}

1. Anuarios de Demografía MINSAL-INE-Registro CivilCELADE, 1960-1989.

2. Informe de un Comisé de Experios de la OMS: Prevención y lucha contra las enfemedades cardiovasculares en la comunidad. Serie de Informes Técnicos $732, \mathbf{G j}$ nebra 1986.
3. Fredrickson DS, Breslow JL: Primary hiperlipoproteinaemia in infants. Annu Ref Med 1973: 24: 315.

4. Milos $C$. Casantuva $V$, Campos $R$, et al.: Factores de riesgo de enfemedad cardiovascular en una poblacion de escolares chilenos. Rev. Chil. Ped. 1990, 61(2): 67.73 .

5. National Cholester of Education Program. Report of the Expert Panel on Blood Cholesterol Levels in Children and Adolescent. Pediatrics 1992; 89: 525 . 584

6. Kallo $M$, Solmenperä $L$, Simes $M$, et al.: Exclusive breast-feeding and weaning: effect on serum choles. terol and lipoprotein concentuations in infants during the first year of life. Pediatrics 1992; 89: 663-666.

7. Strobl W, Widhalm $X$, Kosiner G. Pollak A: Serum apolipoprotein(a) during the first week of life. Acta Paediatr Scand: 1983; 72: 505-509.

8. Darmady J, Fasbrooke A, Lloyd J: Prospective study of serum cholesterul levels during the first year of life. Br Med J 1972; 2: 685-688.

9. Boulton TJ, Craig IH, Hil/ C: Screening of cond blood low density-lipoprotein cholesterot in the diagnosis of familial hypercholesterolaemic a study of 2000 infants Acla Paediat Scand 1979; 68: 363-370.

10. Doniel $M$, Lane J, Mc Connathy $W$ : Factors affecting the lipid and apolipoprotein levels of cord scra. Pediatr Res 1983: 17: 83-91.

11. Hardell $L$ : Serum lipids and lipoproteins at birth based on a stody of 2815 newborn infants. Acra Pacdiatr Scand 1980: Suppl 285: 5-10.

12. Mc Connathy $W$, Lane $D$ : Sudies on the apoliproteins and lipoproteins and lipoprotejns of cord serum. Pediatr Res 1980; 14: 757-761.

13. Biezenski J: Felal lipid metabolism. Obstct Gynecol Annu 1975; 4: 39.

14. Van Bierbliet $J$. Vercaemns $R$, De Keersgieter, et al.: Evolution of lipoprotcin paltems in new borns. Acta Paediatr Scand 1980; Suppl 285: 5-10.

15. Hardefl L. Carison L: Concentration and composition of human senum lipoproteins at birth. Clin Chim Acta 1978; 90: 285-294.

16. Cress $H$, Shaher $R$, Laffin $R$, et al.: Cord blood hyper lipoproteinemia and perinatal suress. Podiatr Res 1992; 31: 396-400.

17. Rudling $M$, Etersen C: C-LDL receptors in bovine tis sues assayed as the heparin sensitive binding of $I$ lebelled in homogenates. Biochem Biophys Act 1985 ; 836: 96-104.

18. Carr $B R$, Simpson $E$ : Lipoprotein utilization and cholesterol synthesis by the human fetal adrenal gland. Endocr Rev 1990; 2: 306-326.

19. Levy RI, Rifkind BM: Diagnosis and management of hiperlipoproteimemia in infants. Amer J Cardiol 1973: 31: 547 . 